\title{
Review
}

\section{Hand-Sewn versus Stapled Closure of Loop Ileostomy: A Meta-Analysis}

\author{
Rana Madani $^{\mathrm{a}}$ Nigel Day ${ }^{\mathrm{b}}$ Lalit Kumar ${ }^{\mathrm{a}}$ Henry S. Tilney ${ }^{\mathrm{a}}$ \\ Andrew Mark Gudgeon ${ }^{\mathrm{a}}$ \\ ${ }^{a}$ Department of Surgery, Frimley Park Hospital, Camberely, UK; ${ }^{b}$ Department of Surgery, Kingston Hospital, \\ Kingston Upon Thames, UK
}

\section{Keywords}

Ileostomy closure $\cdot$ lleostomy reversal $\cdot$ Hand-sewn

anastomosis · Stapled anastomosis

\begin{abstract}
Background: Individual trials comparing hand-sewn with stapled closure of loop ileostomy show different outcomes due to lack of statistical power. A systematic review, with a pooled analysis of results, might provide a more definitive answer. This review aimed to compare hand-sewn with stapled anastomotic technique for closure of a loop ileostomy and looked at the effect of bowel resection on the complication rates. Methodology: Relevant studies were identified from MEDLINE, EMBASE and the Cochrane database. All randomised clinical trials, prospective and retrospective studies comparing hand-sewn with stapled closure of loop ileostomy were included. Results: Of the 4,917 patients in 15 identified studies, 3,406 had hand-sewn and 1,511 stapled anastomosis. There was no difference in the rate of anastomotic leak between the hand-sewn $(2.93 \%, 55 / 1,877)$ and the stapled group $(2.08 \%, 25 / 1,202)$ (OR 0.81, 95\% Cl 0.43-1.54, $p=$ $\left.0.52, I^{2}=33 \%\right)$. The rate of small-bowel obstruction was higher in the hand-sewn group $(7.03 \%, 231 / 3,284)$ compared to the stapled group $(5.58 \%, 73 / 1,308$; OR $0.69,95 \% \mathrm{Cl} 0.51-$ $\left.0.92, p=0.01, R^{2}=0 \%\right)$. There was no difference in the inci-
\end{abstract}

dence of anastomotic leak and small-bowel obstruction in the hand-sewn anastomosis between patients with or without bowel resection. Conclusions: There was no significant difference in the rate of anastomotic leakage between the hand-sewn and stapled techniques. The rate of small-bowel obstruction was higher in the hand-sewn group. Performance of bowel resection does not significantly increase the incidence of anastomotic leak or small-bowel obstruction.

(c) 2018 S. Karger AG, Basel

\section{Background}

A defunctioning loop ileostomy is a commonly used surgical procedure to allow faecal diversion from a distal anastomosis. This technique is primarily used to facilitate the healing of a low colorectal anastomosis to diminish the consequences of an anastomotic leak. The most common conditions where a loop ileostomy may be used include resections for rectal cancer, ulcerative colitis, diverticular disease and familial adenomatous polyposis. It can also be used as a diversion in severe fistulating perianal Crohn's disease. Once the anastomosis has healed or a fistula successfully repaired, the loop ileostomy may be closed [1].

The traditional method for reversing a loop ileostomy involved a hand-sewn end-to-end bowel anastomosis.

\section{KARGER}

(c) 2018 S. Karger AG, Basel

E-Mail karger@karger.com

www.karger.com/dsu
Ms. Rana Madani

Department of Colorectal Surgery

Frimley Park Hospital

Portsmouth Road, Frimley, Surrey GU16 7UJ (UK)

E-Mail RanaMadani@ doctors.org.uk 
The side-to-side stapler anastomosis for closure of ileostomy was introduced into practice in the 1980s [2]. Individual trials comparing hand-sewn with stapled closure of loop ileostomy showed different outcomes, perhaps because they lacked statistical power. A systematic review, with a pooled analysis of results, might provide a more definitive answer.

Although systematic reviews on this topic already exist in the literature, none of them has looked into the effect of small-bowel resection during the ileostomy reversal procedure. This review aims to compare stapled versus hand-sewn anastomoses with regard to all documented parameters of perioperative morbidity in addition to looking at the effects of small-bowel resection on anastomotic leak and bowel obstruction.

\section{Methods}

All published randomised controlled trials and quasi-randomised controlled trials comparing stapled and hand-sewn closure of loop ileostomy were eligible for inclusion. All relevant studies were considered, irrespective of language and publication status, for this review. There were only 4 randomised control trials in the literature. One study was a prospective non-randomised [3] and one was a retrospective analysis of a prospective database [4]. There was only one study with more than 1,000 participants [5].

The primary endpoint was anastomotic leak and the secondary endpoint included bowel obstruction, wound infection, bleeding, operating time, time to first defecation, length of hospital stay, readmission, re-operation and 30 days operative mortality.

\section{Search Strategy}

Systematic search strategies was used to identify the relevant studies and electronic search was performed. The databases used for the literature search included MEDLINE, EMBASE, and Cochrane Controlled Trials Register. The reference lists in the identified studies were also searched in order to identify further studies.

\section{Data Collection}

Two authors independently assessed the titles and abstracts of the identified studies. All full articles were obtained for the included studies. Only studies that had compared stapled and hand-sewn anastomosis, with or without bowel resection, were included in the analysis. All abstracts identified by the above search strategies were assessed for subject relevance. A data abstraction form was developed to record details of study design, participants, year of study, patient characteristics and outcomes.

\section{Statistical Analysis}

Meta-analyses were performed according to the recommendations of the Cochrane Handbook for Systematic Reviews of Interventions. The Mantel-Haenzel's methods were used to perform the meta-analysis with the random-effects model [6].

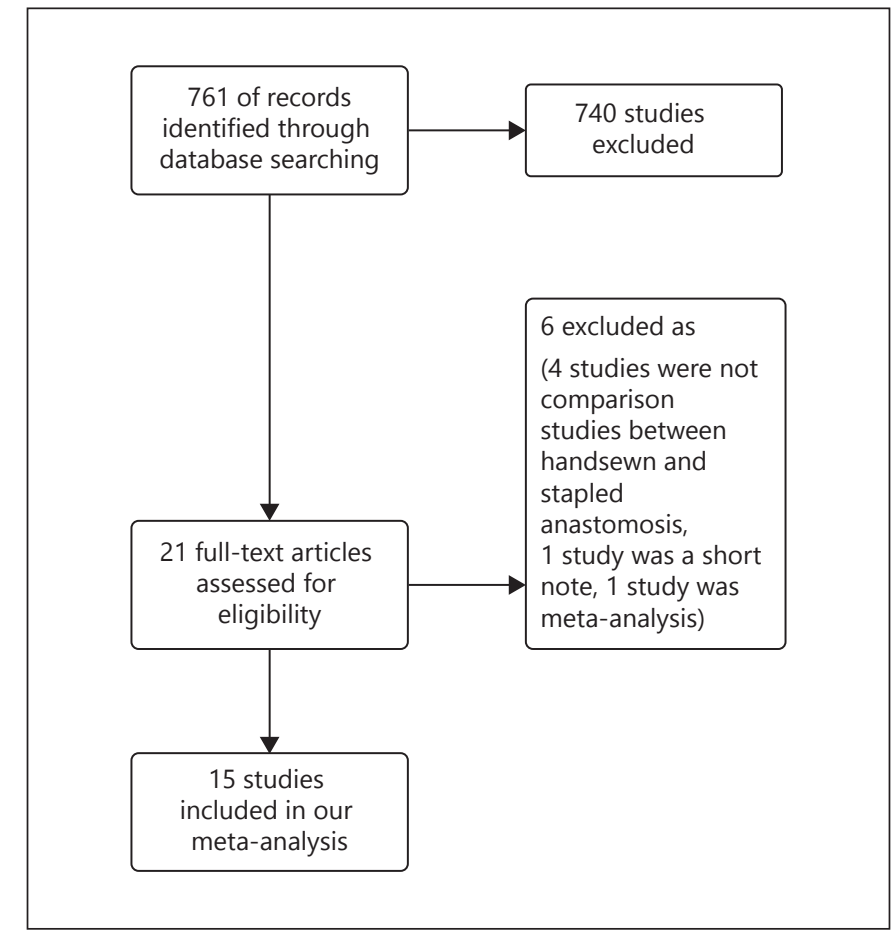

Fig. 1. Selection of articles for meta-analysis.

The software package, RevMan 5.0, was used for this review. For dichotomous variables, the OR and for continuous outcomes, weighted mean difference (WMD) was calculated with $95 \%$ CI and random effect. The heterogeneity was explored using $l^{2}$ and $p$ value from the chi-square test whenever required.

Six studies $[3,4,7-10]$ had sub-divided the hand-sewn and one study the stapled patients [11], into further subgroups of closures with and without bowel resection. In our analysis, these subgroups were amalgamated into one group in each category, although a further subgroup analysis was performed to compare outcomes in patients with and without bowel resection. The mean of the re-grouped data was calculated by using the following formula: ([mean of group $\mathrm{A} \times$ number of group $\mathrm{A})+($ mean group $B \times$ number of group $B]$ )/(number of group $A+$ number of group B).

If only range was provided, $\mathrm{SD}$ was calculated from range by using the following method, as described by Hozo et al. [12]. In a moderate sample size $(15<n \leq 70)$, the best estimator for the standard deviation (variance) was used as $\mathrm{SD}=$ range $/ 4$ and for large samples $(n>70), \mathrm{SD}=$ range/ 6 . In one study [13] where only CI was provided, $\mathrm{SD}$ was calculated using the formula, $\mathrm{CI}=2 \times 1.96 \times$ SD.

Where there was heterogeneity with the SD, the large SD was used. In the studies by Garcia-Botello et al. [3] and Phang et al. [10], the outcome data had to be calculated from the graphs as actual values had not been provided. This was done by 2 authors independently and they were found to be in agreement.

Publication bias was explored through a funnel plot of effect estimates. The asymmetry in the funnel plot of study size was used against effect to identify this bias. 
Table 1. Characteristics of the included studies

\begin{tabular}{|c|c|c|c|c|c|}
\hline Balik et al. [22], 2011 & Clin Sci & 2011 & Retrospective & $\begin{array}{l}2002-2005 \text { open } \\
2005-2007 \text { staple }\end{array}$ & 225 \\
\hline Feinberg et al. [8], 1987 & Am J Surg & 1987 & Retrospective & $1981-1985$ & 110 \\
\hline $\begin{array}{l}\text { Garcia-Botello } \\
\text { et al. [3], } 2004\end{array}$ & Dig Surg & 2004 & $\begin{array}{l}\text { Prospective non } \\
\text { randomised }\end{array}$ & 1992-2002 & 109 \\
\hline Gustavsson et al. [9], 2012 & Int J Colorectal Dis & 2012 & Retrospective & 1999-2006 & 351 \\
\hline Hull et al. [18], 1996 & DCR & 1996 & RCT & 1993-1994 & 61 \\
\hline Löffler et al. [15], 2012 & Ann Surg & 2012 & RCT & 2009-2010 & 324 \\
\hline Luglio et al. [4], 2011 & Arch Surg & 2011 & Retrospective & 2005-2010 & 944 \\
\hline Phang et al. [10], 1999 & Am J Surg & 1999 & Restrospective & 1987-1995 & 339 \\
\hline Saha et al. [16], 2009 & Colorectal Dis & 2009 & Retrospective & 1999-2005 & 325 \\
\hline Shelygin et al. [14], 2010 & Tech Coloprocto & 2010 & RCT & 2005-2008 & 119 \\
\hline Wong et al. [5], 2005 & DCR & 2005 & Retrospective & 1983-2002 & 1,504 \\
\hline
\end{tabular}

Results of the Search

Sixteen relevant papers in total were identified. One was a meta-analysis and therefore 15 studies were included in this review. The study flow diagram is shown below (Fig. 1).

\section{Results}

Fifteen studies met the inclusion criteria after a systematic search. The characteristics of the included trials are summarised in Table 1. Two randomised clinical trials (RCTs) $[14,15]$ described their randomisation technique. The former study also described its blinding technique, but Löffler et al. [15] had not blinded the patients or surgeons. Two papers (Hull et al. [18], Wong et al. [5]) were from the same centre. Of the 2, Wong et al. [5] was a large retrospective analysis of data over 19 years. This study may have included the smaller RCT, which had been performed for over a year (Hull et al. [18]) in the same centre. As there had been outcome measures in the
RCT, which had not been mentioned by Wong et al. [5], we elected to include both studies. This could introduce bias into the analysis; however, the number in the small RCT was significantly smaller than that in the large retrospective analysis and therefore, the bias would be unlikely to have a significant effect on the overall outcome.

Five papers (D'Haeninck et al. [7], Garcia-Botello et al. [3]; Gustavsson et al. [9], Phang et al. [10], Feinberg et al. [8]) divided the hand-sewn group into 2 subgroups; with and without bowel resection. In our analysis, these 2 subgroups were re-grouped under the handsewn categories. An analysis of the subgroups was performed looking at the differences between patients with and without bowel resection. Horisberger et al. [11] divided the patients with stapled anastomosis, into $2 \mathrm{sub-}$ groups; with and without bowel resection. In our metaanalysis, these subgroups were also re-grouped under the common heading of stapled anastomosis.

In a study by Saha et al. [16], 38\% of the patient had their primary operation as an emergency. No other stud- 
Table 2. Patient characteristics of hand-sewn and stapled groups in each included study

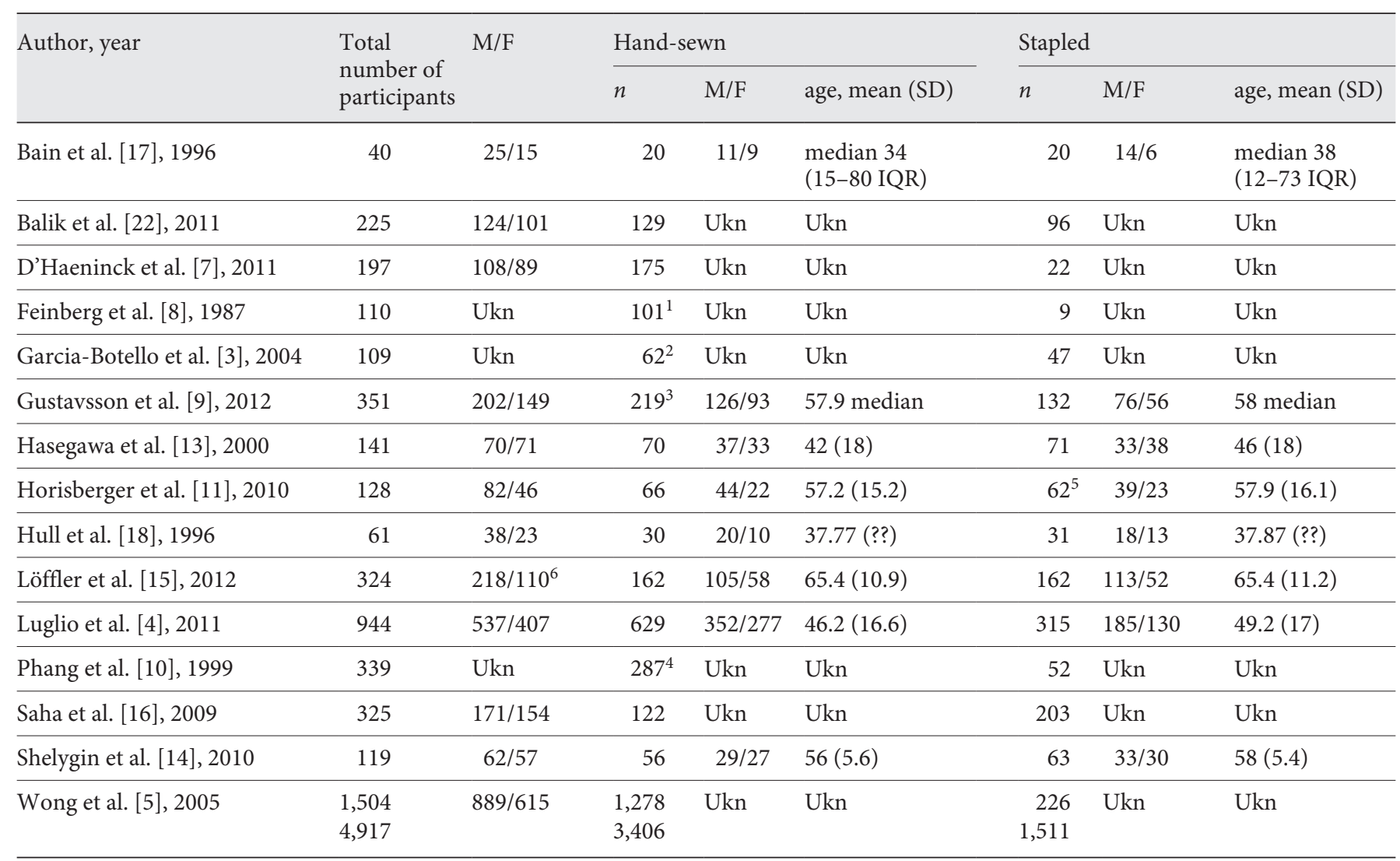

${ }^{1}$ Of the 101 total patients, 27 did and 74 did not have bowel resection. ${ }^{2}$ Of the 62 total patients, 28 did and 34 did not have bowel resection. ${ }^{3}$ Of the 219 total patients, 70 did and 149 did not have bowel resection. ${ }^{4}$ Of the 287 total patients, 220 did and 67 did not have bowel resection. ${ }^{5}$ Of the 62 total patients, 2 groups of stapled with and without resection are merged. ${ }^{6}$ The male/female ratio was provided for 328 patients prior to excluded patients and therefore total adds up to 328 .

ies provided this data and therefore it was not possible to ascertain whether this is an important risk factor in occurrence of future complications. The primary pathology of patients varied widely. Only 2 of the studies (Luglio et al. [4], Bain et al. [17]) provided the breakdown of the patient's pathology for both the hand-sewn and stapled groups. There was therefore not enough information to analyse this any further.

\section{Patient Data}

All 15 papers were reported as full papers and included a total of 4,917 participants. The age and sex characteristics of the study are in Table 2. One study did not provide the sex ratio (Phang et al. [10]) and in 2 other (Feinberg et al. [8], Garcia-Botello et al. [3]) studies, the sex ratio was provided only for the patients who had undergone formation of ileostomy. This information was not given for patients who then subsequently went on to have reversal of their ileostomy. Loffler et al. [19] provide this ratio prior to exclusion of 4 patients. Six studies did not have the sex ratio compared between the 2 hand-sewn and stapled groups (Balik et al. [22], Feinberg et al. [8], Garcia-Botello et al. [3], Phang et al. [10], Saha et al. [16], Wong et al. [5]).

Those in the stapled group were slightly older than those in the hand-sewn group (WMD: 1.79 years, 95\% CI $0.58-3.00, p=0.004, I^{2}=0 \%$ ). This difference was only by under 2 years, but it was statistically significant.

\section{Complications Data}

Of the total 4,917 participants, 3,406 underwent hand-sewn and 1,511 underwent stapled anastomosis. Table 3 shows a summary of the complication and outcome data. 
Table 3. Complications and outcome data

\begin{tabular}{llll}
\hline Outcomes & $\begin{array}{l}\text { Number } \\
\text { of studies }\end{array}$ & $\begin{array}{l}\text { Number } \\
\text { of participants }\end{array}$ & OR or WMD \\
\hline Anastomotic leak & 12 & 3,079 & OR 0.81, 95\% CI 0.43-1.54 \\
Small bowel obstruction & 14 & 4,592 & OR 0.69, 95\% CI 0.51-0.92 \\
Small bowel obstruction and ileus & 13 & 4,268 & OR 0.61, 95\% CI 0.43-0.86 \\
Wound infection & 10 & 3,595 & OR 1.44, 95\% CI 0.94-2.20 \\
Anastomotic haemorrhage & 7 & 3,500 & OR 4.12, 95\% CI $1.29-13.18$ \\
Operative time & 8 & 1,954 & WMD -10.18 min, $95 \%$ CI -16.55 to -3.82 \\
Time to first bowel movement & 6 & 1,801 & WMD -0.35 days, $95 \%$ CI -0.57 to -0.14 \\
Length of stay & 8 & 3,425 & WMD -1.17 days, $95 \%$ CI -1.70 to -0.64 \\
Re-operating & 8 & 2,087 & OR 0.93, 95\% CI 0.58-1.48 \\
Re-admission & 4 & 1,265 & OR 0.72, 95\% CI $0.38-1.37$ \\
\hline
\end{tabular}

Dichotomous data were analysed with OR and continuous data with WMD, with 95\% CI and random-effect model.

\section{Anastomotic Leak}

Seven studies employed differing definitions of anastomotic leak (D'Haeninck et al. [7], Garcia-Botello et al. [3], Gustavsson et al. [23], Hasegawa et al. [13], Horisberger et al. [11], Luglio et al. [4], Saha et al. [16]). Four authors defined leak as when there was radiological evidence and/or operative signs of leak (Hasegawa et al. [13], Gustavsson et al. [9], Saha et al. [16], Garcia-Botello et al. [3]). Horisberger et al. [11] defined leak as when there was radiological with clinical symptoms that lead to re-operation. Eight studies did not provide any definition (Bain et al. [17], Balik et al. [22], Feinberg et al. [8], Löffler et al. [15], Shelygin et al. [14], Wong et al. [5], Hull et al. [18], Phang et al. [10]). Wong et al. [5] did not describe leak as a separate entity but as part of a wider heading of anastomotic complications and therefore, it was not possible to calculate their leak rate. Bain et al. [17], however, reported one fistula in the hand-sewn group, a complication that may have been a manifestation of an anastomotic leak.

The anastomotic leak rate was $2.93 \%(55 / 1,877)$ in the hand-sewn and $2.08 \%(25 / 1,202)$ in the stapled group. There was no difference between the 2 techniques as seen by the forest plot (OR $0.81,95 \%$ CI $0.43-1.54, p=0.52, I^{2}$ $=33 \%$; Fig. 2).

Luglio et al. [4] considered abdominal abscess collection in a different category. Although this could have been considered to be a manifestation of an anastomotic leak, authors specify that an anastomotic dehiscence had been excluded either at abdominal exploration or with abdominal imaging. To exclude selection bias, we aimed to analyse the data of randomised control trials only. However, one RCT had no leak in their cohort of patients (Hull et al. [18]) and a second RCT (Shelygin et al. [14]) had not reported their leak data. Nevertheless, analysis on the basis of only 2 RCTs confirmed no difference in the anastomotic leak rate between the 2 techniques (OR 0.87, $95 \%$ CI 0.12-6.33, $p=0.89, R^{2}=39 \%$; Fig. 3 ).

In order to evaluate the effect of bowel resection, a subgroup analysis was performed and anastomotic leak rate was compared between patients with and without bowel resection in the hand-sewn group. There no was no difference in the rate of anastomotic leak between the 2 groups (OR 0.78, 95\% CI 0.33-1.87, $p=0.58, R^{2}=0 \%$; Fig. 4 ).

\section{Small-Bowel Obstruction}

Ten studies had defined bowel obstruction (D'Haeninck et al. [7], Feinberg et al. [8], Garcia-Botello et al. [3], Gustavsson et al. [9], Hasegawa et al. [13], Löffler et al. [15], Luglio et al. [4], Phang et al. [10], Saha et al. [16], Wong et al. [5]). All except, one agree with the description for bowel obstruction as being when clinical and radiological signs suggest bowel obstruction. Hasegawa et al. [13] and Garcia-Botello et al. [3] state that this should have been within 30 days. Luglio et al. [4] however would consider bowel obstruction only if it needed re-operation. The forest plot of the data (Fig. 5) shows that small-bowel obstruction is more likely to occur in hand-sewn $(7.03 \%, 231 / 3,284)$ compared to stapled $(5.58 \%, 73 / 1,308)$ anastomosis (OR $0.69,95 \%$ CI $0.51-0.92, p=0.01, P^{2}=0 \%$; Fig. 5).

Analysis of the 4 RCTs found this difference to be equally significant in favour of stapled anastomosis (OR $0.54,95 \%$ CI $0.30-0.95, p=0.03, P^{2}=0 \%$; Fig. 6).

Horisberger et al. [11] did not report any obstruction, but ileus was reported in 8 patients ( 4 in each group). Ileus was defined as a delay in feeding of $>5$ days or the requirement for a nasogastric tube. Luglio et al. [4] grouped 


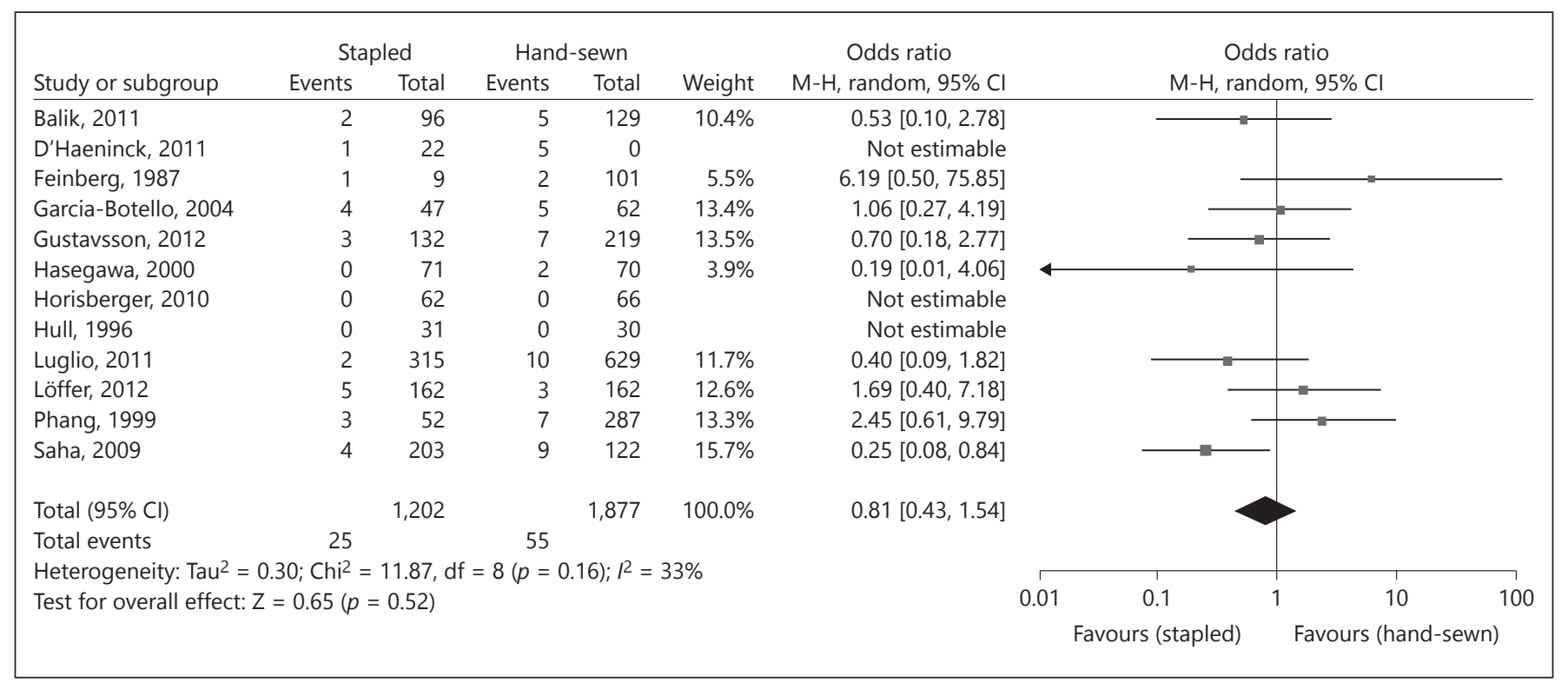

Fig. 2. Forest plot showing OR in individual studies and overall pooled OR evaluating anastomotic leak.

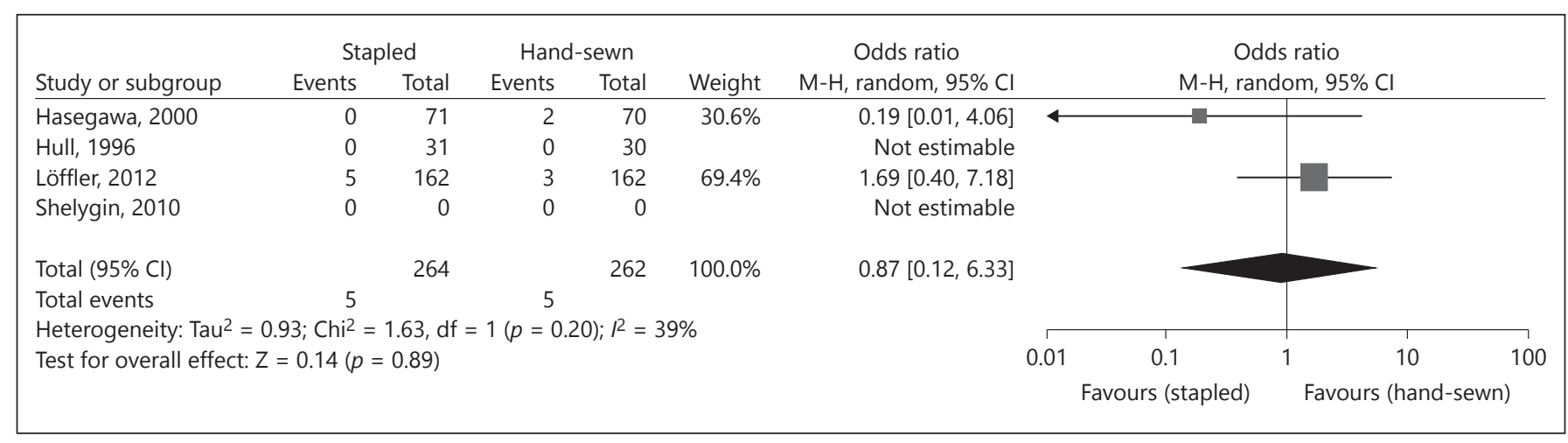

Fig. 3. Forest plot showing OR in individual studies and overall pooled OR in the 2 RCTs, which reported anastomotic leaks.

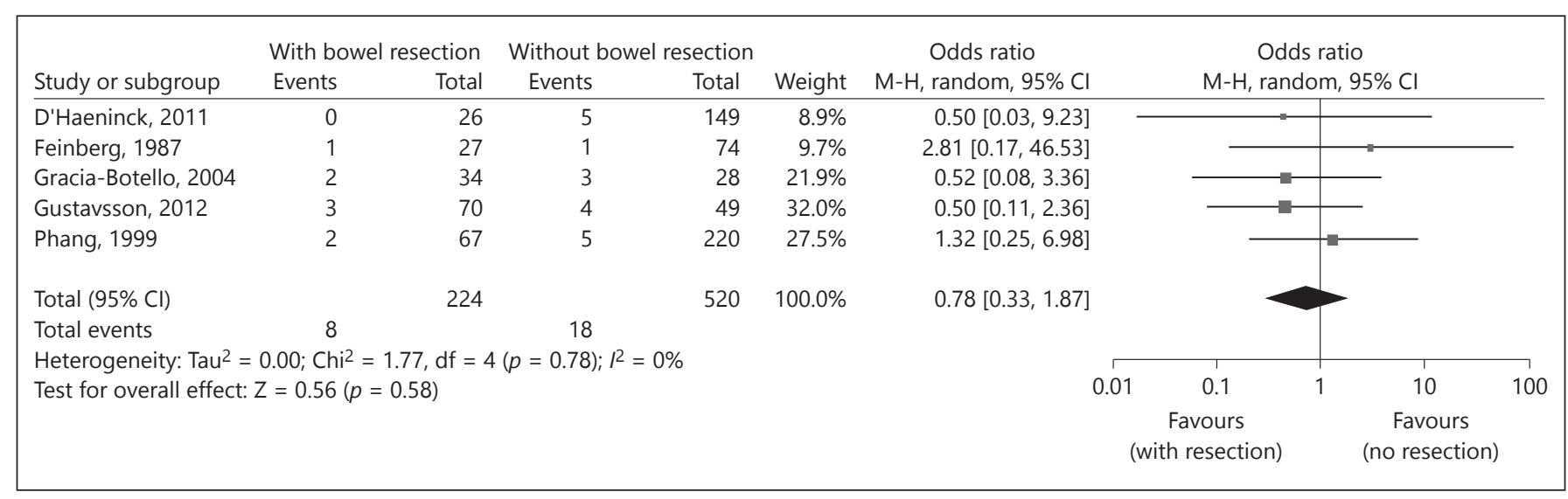

Fig. 4. Forest plot showing OR in individual studies and overall pooled OR evaluating the effect of small-bowel resection on anastomotic leaks. 


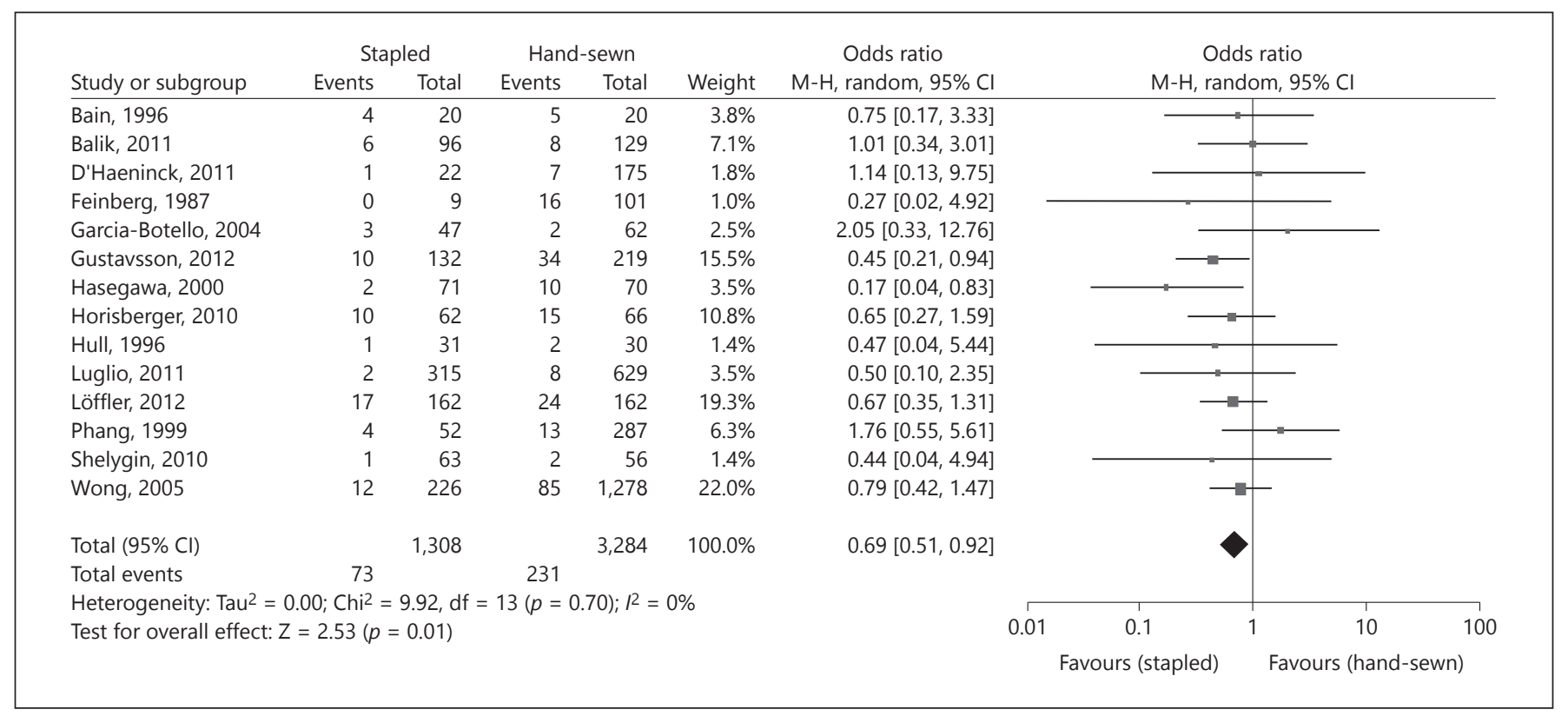

Fig. 5. Forest plot showing OR in individual studies and overall pooled OR for small-bowel obstruction.

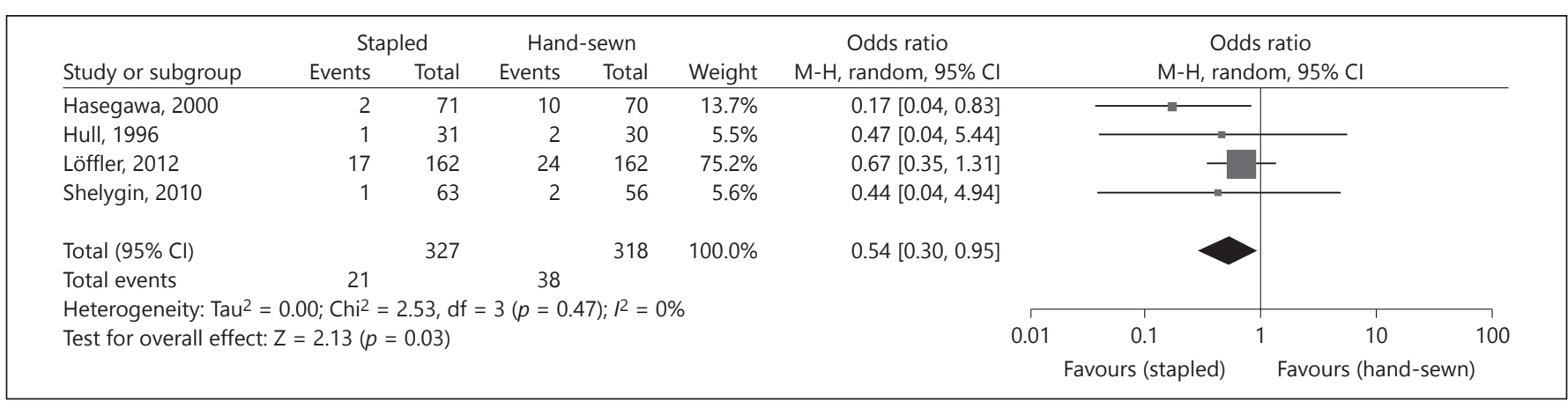

Fig. 6. Forest plot showing OR for small-bowel obstruction in 4 RCTs and the overall pooled OR.

all patients who did not need re-operation as ileus or partial small-bowel obstruction (99 in hand-sewn and 21 in stapled). As definitions of bowel obstruction varied, in some studies, patients with bowel obstruction could have been labelled as having had ileus or vice versa and this could have introduced bias in the analysis. An analysis of all patients with small-bowel obstruction and ileus shows this difference to be even more significant in favour of stapled anastomosis (OR 0.61, 95\% CI 0.43-0.86, $p=$ $0.006, R^{2}=29 \%$; Fig. 7 ).

Reduced incidence of small-bowel obstruction with stapled anastomosis could be explained by the fact that the calibre of the bowel lumen could be much smaller with hand-sewn techniques and, in general, far wider with stapled anastomosis. As a consequence, it is more

Hand-Sewn versus Stapled Closure of Loop Ileostomy: A Meta-Analysis likely that perioperative oedema might compromise the luminal diameter enough to cause early bowel obstruction with the former method [1].

A subgroups analysis of hand-sewn anastomosis comparing ones with and without bowel resection showed no difference between the 2 groups (OR 1.06, 95\% CI $\left.0.38-2.93, p=0.91, l^{2}=63 \%\right)$. However, the numbers in this subgroup analysis are small with significant heterogeneity (Fig. 8).

\section{Wound Infection and Breakdown}

Four authors (D'Haeninck et al. [7], Hasegawa et al. [13], Phang et al. [10], Wong et al. [5]) defined wound infection by the presence of erythema and pus discharge. Saha et al. [16] reported a wound as infected when ery- 


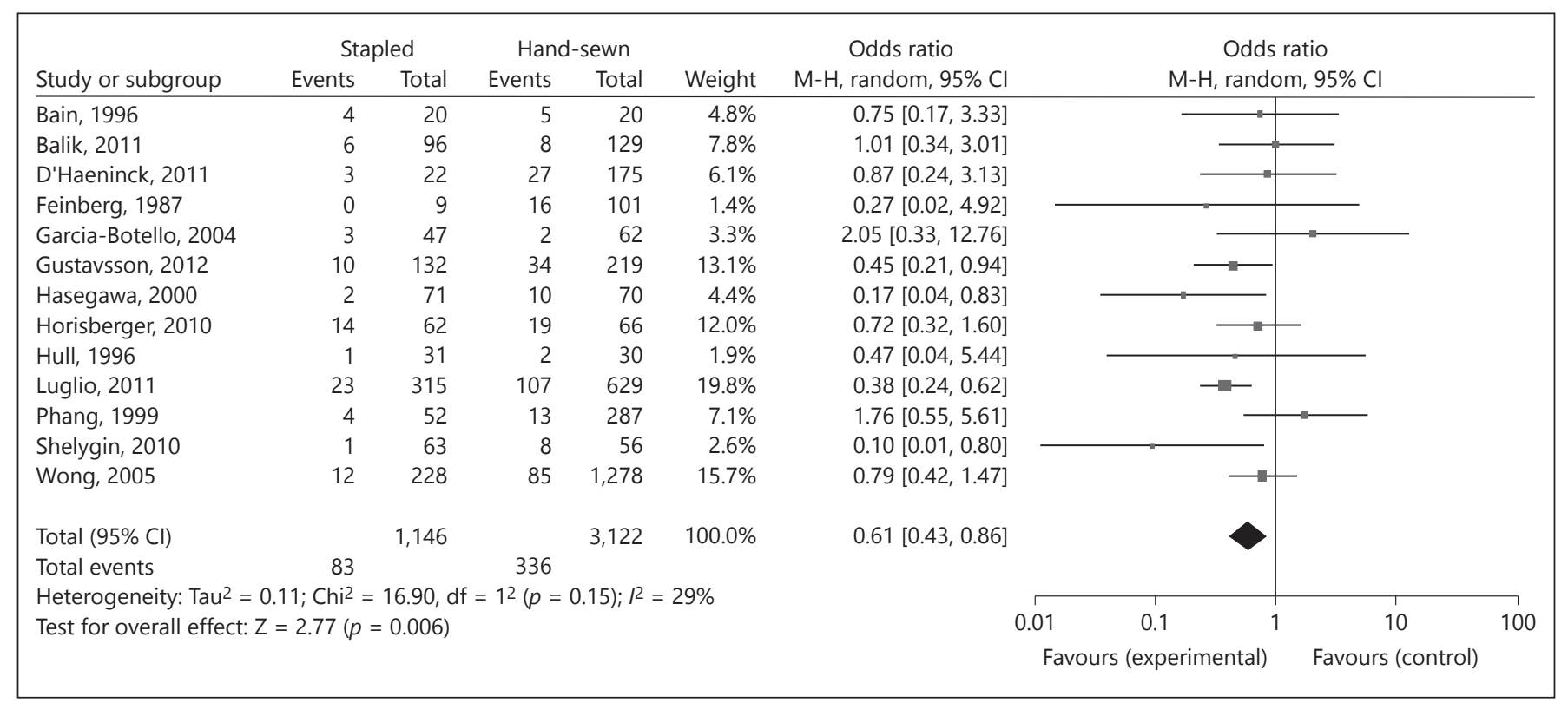

Fig. 7. Forest plot showing individual and overall pooled OR for ileus and small-bowel obstruction.

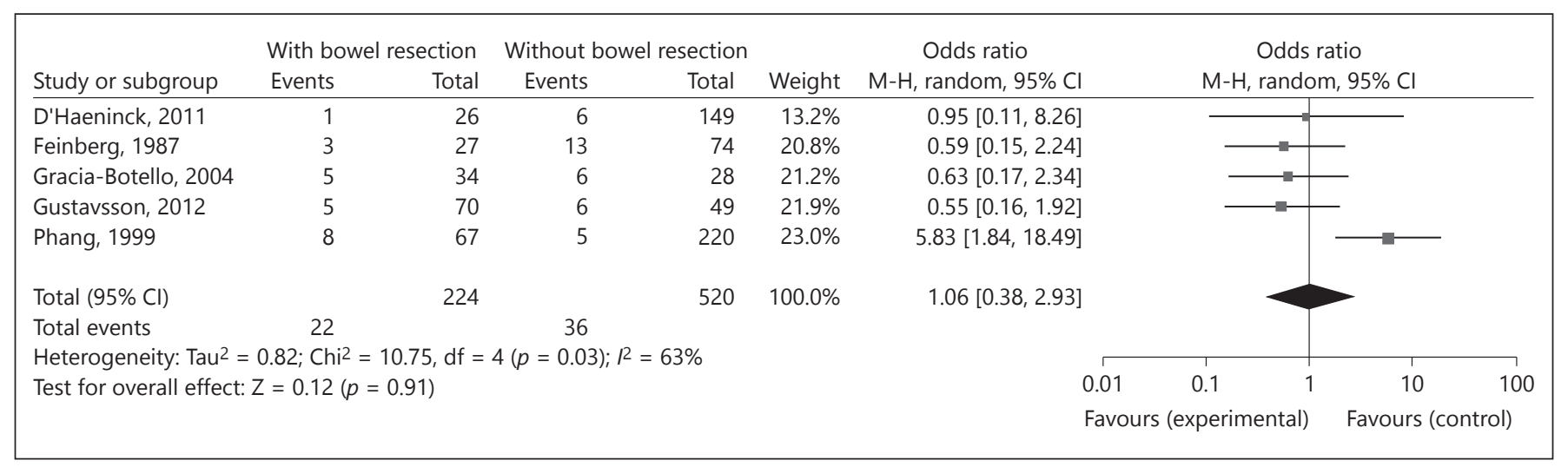

Fig. 8. Forest plot showing individual and overall pooled OR evaluating effect of bowel resection on small-bowel obstruction.

thema was associated with positive swab culture and Hoisberger et al. [11] followed the criteria of disease control and prevention. The rate of wound infection was $3.44 \%(86 / 2,502)$ in hand-sewn and $7.5 \%(82 / 1,093)$ in the stapled anastomosis group. There was no statistical difference in the rate of wound infection between the 2 groups (OR 1.44, 95\% CI 0.94-2.20, $p=0.09, l^{2}=26 \%$; Fig. 9).

Also, analysis of RCTs only found no difference between the 2 groups in the rate of wound infection (OR $1.16,95 \%$ CI $0.67-1.99, p=0.60, I^{2}=0 \%$ ).

The presence of wound infection could have been influenced by the technique of skin closure. However, 4 au- thors closed the skin (Bain et al. [17], Garcia-Botello et al. [3], Hasegawa et al. [13], Horisberger et al. [11]), 2 left the skin open/partially open (Hull et al. [18], Wong et al. [5]), 2 approximated the skin with interrupted sutures (D'Haeninck et al. [7], Löffler et al. [15]) and others did not elaborate on their skin closure techniques. The potential role of wound closure techniques in wound infection after ileostomy closure requires further study.

\section{Anastomotic Haemorrhage}

Anastomotic haemorrhage was reported in 7 studies (Hasegawa et al. [13], Horisberger et al. [11], Luglio et al. [4], Phang et al. [10], Saha et al. [16], Shelygin et al. [14], 


\begin{tabular}{|c|c|c|c|c|c|c|c|c|c|c|}
\hline \multirow{3}{*}{$\frac{\text { Study or subgroup }}{\text { Bain, } 1996}$} & \multicolumn{2}{|c|}{ Stapled } & \multicolumn{2}{|c|}{ Hand-sewn } & \multirow[b]{2}{*}{ Weight } & \multirow{2}{*}{$\begin{array}{c}\text { Odds ratio } \\
\mathrm{M}-\mathrm{H} \text {, random, } 95 \% \mathrm{Cl}\end{array}$} & \multirow{2}{*}{\multicolumn{4}{|c|}{$\begin{array}{c}\text { Odds ratio } \\
\mathrm{M}-\mathrm{H}, \text { random, } 95 \% \mathrm{Cl}\end{array}$}} \\
\hline & Events & Total & Events & Total & & & & & & \\
\hline & 1 & 20 & 2 & 20 & $2.8 \%$ & $0.47[0.04,5.69]$ & & & & \\
\hline Balik, 2011 & 9 & 96 & 7 & 129 & $12.5 \%$ & $1.80[0.65,5.03]$ & & & & \\
\hline D'Haeninck, 2011 & 0 & 0 & 0 & 0 & & Not estimable & & & & \\
\hline Garcia-Botello, 2004 & 9 & 47 & 11 & 62 & $13.5 \%$ & $1.10[0.41,2.91]$ & & & & \\
\hline Hasegawa, 2000 & 6 & 71 & 7 & 70 & $10.6 \%$ & $0.83[0.26,2.61]$ & & & & \\
\hline Horisberger, 2010 & 1 & 62 & 1 & 66 & $2.2 \%$ & $1.07[0.07,17.41]$ & & & & \\
\hline Hull, 1996 & 0 & 31 & 0 & 30 & & Not estimable & & & & \\
\hline Luglio, 2011 & 27 & 315 & 17 & 629 & $23.3 \%$ & $3.38[1.81,6.29]$ & & & 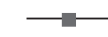 & \\
\hline Löffler, 2012 & 25 & 162 & 19 & 162 & $22.6 \%$ & $1.37[0.72,2.61]$ & & & & \\
\hline Shelygin, 2010 & 1 & 63 & 2 & 56 & $2.9 \%$ & $0.44[0.04,4.94]$ & & & & \\
\hline Wong, 2005 & 3 & 226 & 20 & 1,278 & $9.6 \%$ & $0.85[0.25,2.87]$ & & & & \\
\hline Total $(95 \% \mathrm{Cl})$ & & 1,093 & & 2,502 & $100.0 \%$ & $1.44[0.94,2.20]$ & & & & \\
\hline Total events & 82 & & 86 & & & & & & & \\
\hline \multicolumn{7}{|c|}{ Heterogeneity: Tau $^{2}=0.10 ; \mathrm{Chi}^{2}=10.77, \mathrm{df}=8(p=0.22) ; I^{2}=26 \%$} & 1 & & & 1 \\
\hline \multicolumn{7}{|c|}{ Test for overall effect: $Z=1.67(p=0.09)$} & 0.05 & 0.2 & 5 & 20 \\
\hline & & & & & & & \multicolumn{2}{|c|}{ Favours (stapled) } & avours (ha & ewn) \\
\hline
\end{tabular}

Fig. 9. Forest plot showing individual and overall pooled OR for wound infection.

Wong et al. [5]). Hasegawa et al. [13] reported bleeding when there was continuous intra-abdominal blood loss requiring re-operation and Shelygin et al. [14] had one patient with bloody stool and a drop of $\mathrm{HgB}$. Forest plot analysis shows that the bleeding is significantly more common after stapled anastomosis (OR 4.12, 95\% CI $\left.1.29-13.18, p=0.02, P^{2}=0 \%\right)$. The total incidence, however, is low and in this meta-analysis, only $1.0 \%(10 / 992)$ of the stapled and $0.08 \%(2 / 2,508)$ of the hand-sewn group had anastomotic haemorrhage (Fig. 10).

\section{Operating Time}

Operating time was significantly shorter in stapled anastomosis (WMD $-10.18 \mathrm{~min}, 95 \% \mathrm{CI}-16.55$ to -3.82 , $p=0.002, I^{2}=92 \%$; Fig. 11). It was not possible to analyse the association between operative time and the seniority of the surgeon from the data provided in the studies. This could explain the significant heterogeneity $\left(I^{2}=94 \%\right)$ of the analysis. Operating time was described only in one study, by Hull et al. [18], and therefore, the exact start and finish times may have varied between different studies. The centre's caseload and experience may have been another factor introducing bias in these data.

\section{Time to First Bowel Movement and Length of Stay}

Time to first bowel movement was significantly shorter in the stapled group. Although the time difference was very small (WMD -0.35 days), it was statistically significant $(p=0.001$; Fig. 12$)$.

There was over one day shorter hospital stay in patients with stapled anastomosis and this difference was also statistically significant (WMD -1.17 days, 95\% CI -1.70 to $-0.64, p<0.0001, R^{2}=43 \%$; Fig. 13 ). This would be in keeping with lower rate small-bowel obstruction in this group and consequently shorter time to first bowel movement.

\section{Re-Admission and Re-Operation}

The reason for re-operation was not made clear in all studies. Of the known causes, small-bowel obstruction and anastomotic leak were the most common reason. This was followed by one case of enterotomy [18], which was reported to have been remote from the anastomosis site and so implying that it had not been an anastomotic leak. Anastomotic haemorrhage was the other reason for re-operating, which occurred after a stapled anastomosis [13]. There was, however, no difference in the rate of reoperating between the 2 groups (OR 0.93, 95\% CI $0.58-$ $1.48, p=0.74, R^{2}=0 \%$ ).

There were no differences in the rate of re-admission between the 2 groups (OR $0.72,95 \%$ CI $0.38-1.37, p=$ $0.32, R^{2}=0 \%$ ).

\section{Mortality}

The total 30 days operative mortality in the 8 studies that reported mortality was $0.44 \%(13 / 2,947)$. Studies did not make it clear, however, to which subgroup the deceased patients belonged to. Two studies did not have any case of mortality (Hull et al. [18], Shelygin et al. [14]). Four deaths were as a consequence of an anastomotic leak (D'Haeninck et al. [7], Saha et al. [16]). Deaths were due to 5 myocardial infarction/cardiac events (Phang et al. [10], 


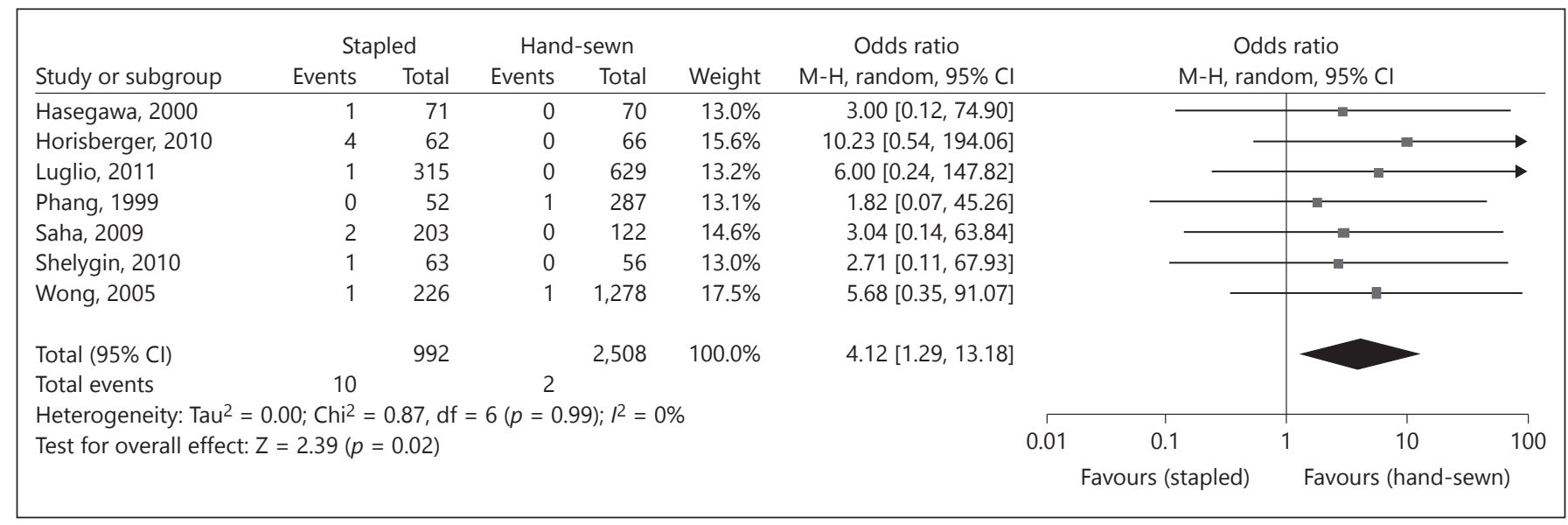

Fig. 10. Forest plot showing individual and overall pooled OR for anastomotic haemorrhage.

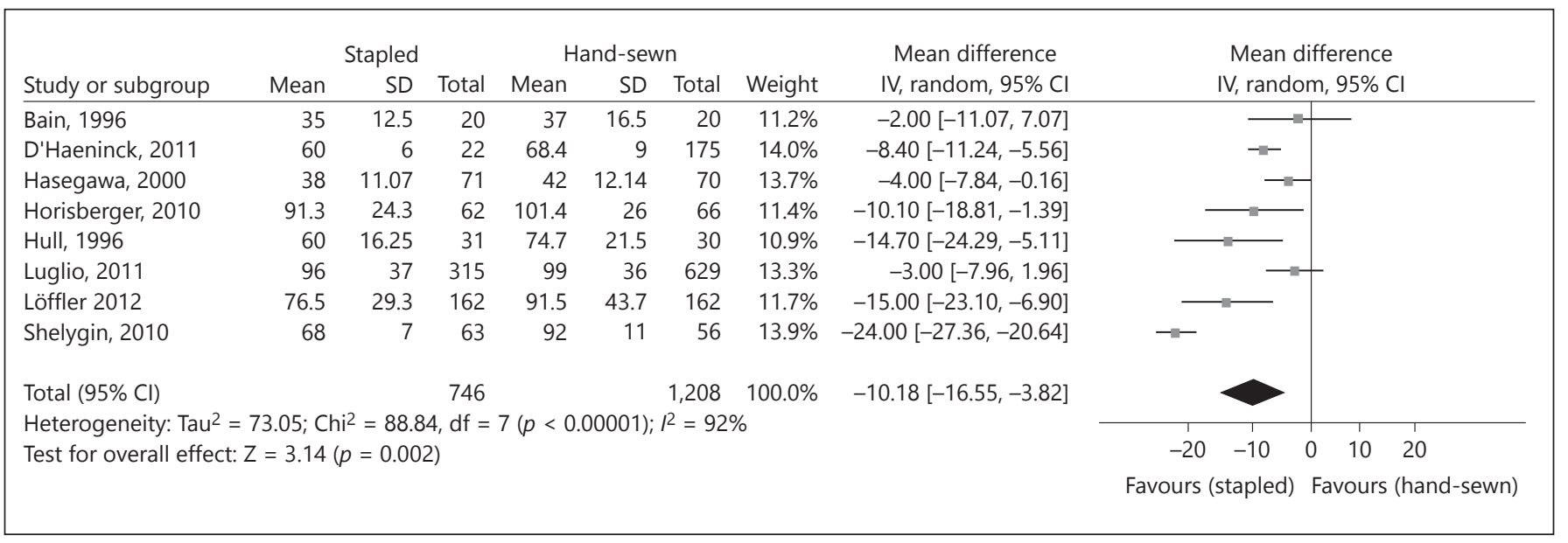

Fig. 11. Forest plot showing individual and overall pooled mean difference in operating time.

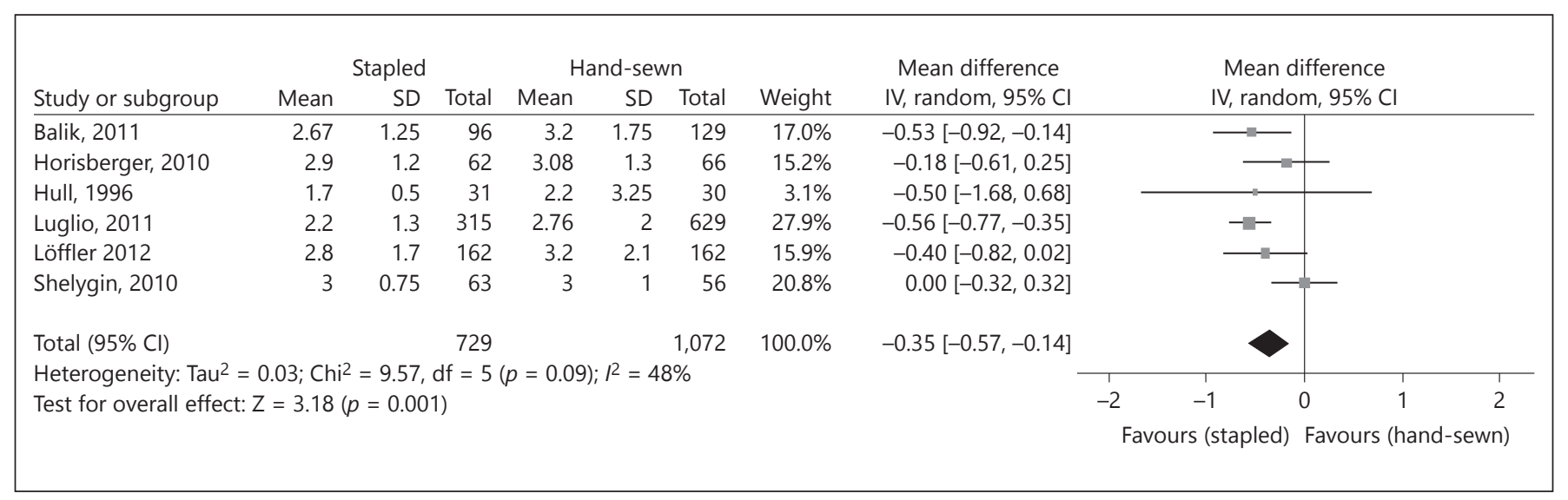

Fig. 12. Forest plot showing individual and overall pooled mean difference in time to first bowel movement. 


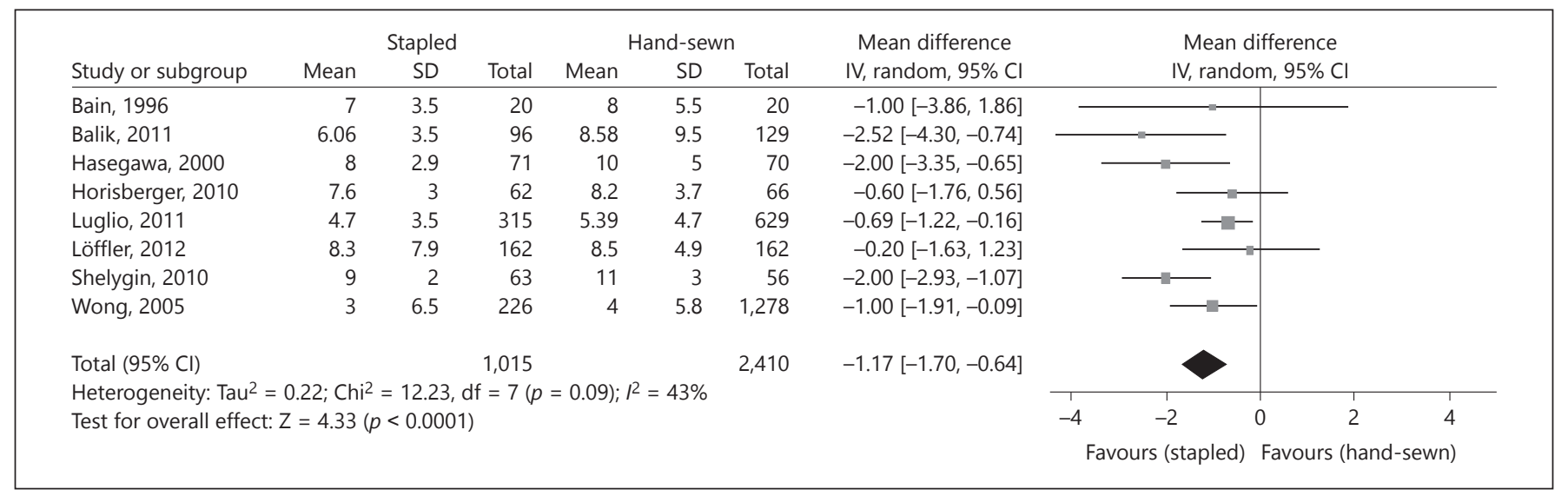

Fig. 13. Forest plot showing individual and overall pooled mean difference in length of stay.

Löffler et al. [15], Saha et al. [16], Wong et al. [5]), one pulmonary embolism (Garcia-Botello et al. [3]), one pneumonia and 2 clostridium difficile events (Saha et al. [16]).

\section{Discussion}

This meta-analysis of 15 studies included all the 4 randomised controlled trials that have been done comparing hand-sewn to stapled anastomosis (Hasegawa et al. [13], Hull et al. [18], Löffler et al. [15], Shelygin et al. [14]). Patients in the stapled groups were less than 2 years older than those in the hand-sewn group. The primary pathology of patients varied widely. Only 2 of the studies (Bain et al. [17], Luglio et al. [4]) provided the breakdown of this data for both the hand-sewn and stapled groups. There was therefore not enough information to analyse this any further.

Anastomotic leak is the most dreaded complication and this review did not demonstrate a significant difference in the leak rate between the hand-sewn and stapled technique. This relates well with previous reviews that also reported similar results. The subgroup analysis looking at the effect of bowel resection on the leak rates did not demonstrate a significant difference between patients with and without bowel resection in the hand-sewn group.

Small-bowel obstruction was significantly more common in the hand-sewn anastomosis. This can possibly be explained by the small calibre of the hand-sewn anastomosis in addition to perioperative oedema leading to a further reduction of the luminal area at the anastomosis [1]. Bowel resection did not seem to increase the risk of small-bowel obstruction significantly. However, the numbers were small in addition to a significant heteroge- neity and we would advise that this result should be interpreted with caution.

Operating time, time to first bowel movement and length of hospital stay were significantly shorter in stapled anastomosis. These are attractive in economic terms and one can argue that these help negate the cost of using a stapler. However, it must be added that operating time did show significant heterogeneity and therefore, the results should be interpreted with caution.

Hand-sewn anastomosis group had a lower risk of anastomotic haemorrhage, although the incidence was small and therefore not likely to influence the overall complication rate significantly. Overall, there were no differences in the rate of re-operation or re-admission between the 2 groups. Small-bowel obstruction and anastomotic leak were the most common reasons for re-operation. The mortality rate in closure of ileostomy was found to be very low $(0.44 \%)$, making this a safe operation.

The results of this study corroborate well with the previous similar studies, which were also unable to detect significant differences between the hand-sewn and the stapled anastomosis [1,19-21]. However, it must be stated that none of the previous studies looked at the effect of small-bowel resection, which was one of the parameter considered in this study. One of the limitations of this study is that some of the studies included are quite old. However, in view of the fact that studies in the last decade have not shown any significant change in the trend, exclusion of such studies would not have made much difference to the results.

\section{Disclosure Statement}

The authors have no conflicts of interest to disclose. 


\section{References}

1 Leung TT, MacLean AR, Buie WD, Dixon E: Comparison of stapled versus handsewn loop Ileostomy closure: a meta-analysis. J Gastrointest Surg 2008;12:939-944.

2 Kestenberg A, Becker JM: A new technique of loop ileostomy closure after endorectal ileoanal anastomosis. Surgery 1985;98:109-111.

3 Garcia-Botello SA, Garcia-Armengol J, Garcia-Granero E, et al: A prospective audit of the complications of loop ileostomy construction and takedown. Dig Surg 2004;21:440-446.

4 Luglio G, Pendlimari R, Holubar SD, Cima RR, Nelson H: Loop ileostomy reversal after colon and rectal surgery: a single institutional 5-year experience in 944 patients. Arch Surg 2011;146:1191-1196.

5 Wong KS, Remzi FH, Gorgun E, et al: Loop ileostomy closure after restorative proctocolectomy: outcome in 1,504 patients. Dis Colon Rectum 2005;48:243-250.

6 DerSimonian R, Laird N: Meta-analysis in clinical trials. Control Clin Trials 1986;7:177188.

7 D'Haeninck A, Wolthuis AM, Penninckx F, D’Hondt M, D’Hoore A: Morbidity after closure of a defunctioning loop ileostomy. Acta Chir Belg 2011;111:136-141.

8 Feinberg SM, McLeod RS, Cohen Z: Complications of loop ileostomy. Am J Surg 1987; 153:102-107.
9 Gustavsson K, Gunnarsson U, Jestin P: Postoperative complications after closure of a diverting ileostoma - differences according to closure technique. Int J Colorectal Dis 2012; 27:55-58.

10 Phang PT, Hain JM, Perez-Ramirez JJ, Madoff RD, Gemlo BT: Techniques and complications of ileostomy takedown. Am J Surg 1999; 177:463-466.

11 Horisberger K, Beldi G, Candinas D: Loop ileostomy closure: comparison of cost effectiveness between suture and stapler. World J Surg 2010;34:2867-2871.

12 Hozo SP, Djulbegovic B, Hozo I: Estimating the mean and variance from the median, range, and the size of a sample. BMC Med Res Methodol 2005;5:13.

13 Hasegawa H, Radley S, Morton DG, Keighley MR: Stapled versus sutured closure of loop ileostomy: a randomized controlled trial. Ann Surg 2000;231:202-204.

14 Shelygin YA, Chernyshov SV, Rybakov EG: Stapled ileostomy closure results in reduction of postoperative morbidity. Tech Coloproctol 2010;14:19-23.

15 Löffler T, Seiler CM, Rossion I, et al: Handsuture versus stapling for closure of loop ileostomy: HASTA-trial: a study rationale and design for a randomized controlled trial. Trials $2011 ; 12: 34-34$
16 Saha AK, Tapping CR, Foley GT, et al: Morbidity and mortality after closure of loop ileostomy. Colorectal Dis 2009;11:866-871.

17 Bain IM, Patel R, Keighley MR: Comparison of sutured and stapled closure of loop ileostomy after restorative proctocolectomy. Ann R Coll Surg Engl 1996;78:555-556.

18 Hull TL, Kobe I, Fazio VW: Comparison of handsewn with stapled loop ileostomy closures. Dis Colon Rectum 1996;39:1086-1089.

19 Loffler T, Rossion I, Goossen K, et al: Hand suture versus stapler for closure of loop ileostomy - a systematic review and meta-analysis of randomized controlled trials. Langenbecks Arch Surg 2015;400:193-205.

20 Markides GA, Wijetunga IU, Brown SR, Anwar S: Meta-analysis of handsewn versus stapled reversal of loop ileostomy. ANZ J Surg 2015;85:217-224.

21 Gong J, Guo Z, Li Y, et al: Stapled vs hand suture closure of loop ileostomy: a meta-analysis. Colorectal Dis 2013;15:e561-e568.

22 Balik E, Eren T, Bugra D, Buyukuncu Y, Akyuz A, Yamaner S: Revisiting stapled and handsewn loop ileostomy closures: a large retrospective series. Clinics 2011;66:1935-1941.

23 Gustavsson K, Gunnarsson U, Jestin P: Postoperative complications after closure of a diverting ileostomy - differences according to closure technique. Int J Colorectal Dis 2012;27:55-58. 